\title{
Evaluation of Pedestrian/Bicycle Crash Injury Case Definitions for Use with NC DETECT
}

\author{
Katherine J. Harmon 1, 2, 3, Amy Ising ${ }^{3}$, Laura Sandt ${ }^{1,}$ 2, Anna E. Waller ${ }^{3}$ \\ ${ }^{1}$ Highway Safety Research Center, Chapel Hill, North Carolina, United States, ${ }^{2}$ Collaborative Sciences Center for Road Safety, Chapel Hill, North \\ Carolina, United States, ${ }^{3}$ Carolina Center for Health Informatics, UNC School of Medicine, Chapel Hill, North Carolina, United States \\ Objective
}

To evaluate four ICD-10-CM based case definitions designed to capture pedestrian and bicycle crash-related emergency department (ED) visits in North Carolina's statewide syndromic surveillance system, NC DETECT.

\section{Introduction}

Over the last few decades, the United States has made considerable progress in decreasing the incidence of motor vehicle occupants injured and killed in traffic collisions [1]. However, there is still a need for continued motor vehicle crash (MVC) injury surveillance, particularly for vulnerable road users, such as pedestrians and bicyclists. In NC, the average annual number of pedestrian-motor vehicle crashes increased by 13.5 percent during the period 2011-2015, as compared to 2006-2010 [2]. Therefore, the Carolina Center for Health Informatics (CCHI), as part of a NC Governor's Highway Safety Program-funded project to improve statewide MVC injury surveillance, developed and evaluated four ICD-10-CM based case definitions for use with NC DETECT, NC's statewide syndromic surveillance system.

\section{Methods}

We created four pedestrian/bicycle crash injury case definitions based on ICD-10-CM transportation codes ("V-codes"): TrafficRelated Pedestrian Crashes, Traffic/Non-Traffic-Related Pedestrian Crashes, Traffic-Related Bicycle Crashes, and Traffic/NonTraffic-Related Bicycle Crashes. These definitions were based on the Centers for Disease Control and Prevention (CDC) "ICD10-CM External Cause of Injury Codes". [3] We then applied these pedestrian/bicycle crash case definitions to 2016-2017 NC DETECT ED visit data and data obtained from a single NC Level I Trauma Center. Next, we linked the two data sources using the variables date of visit, time of visit, and medical record number. Since trauma center data are collected and verified by a designated trauma registrar, we considered the data obtained from the Level I Trauma Center to be the "gold standard".

\section{Results}

The linkage between the two data sources was successful, with $99.5 \%$ of all Level I Trauma Center records linking to ED visits in NC DETECT. However, we found discrepancies in the assignment of codes between the ED visit and Trauma Center data. For example, $47.5 \%$ of NC DETECT ED visits that linked to a pedestrian/bicycle crash record in the Trauma Center data, were missing an ICD-10-CM injury mechanism code of any category. Historically, the proportion of injury-related ED visits that were missing corresponding injury mechanism codes was low $(<15 \%)$. However, the transition from ICD-9-CM to ICD-10-CM increased the proportion of injury-related visits missing injury mechanism codes [4]. Among the 92 NC DETECT ED visits missing injury mechanism codes, $35.9 \%$ contained a pedestrian/bicycle crash-related keyword in the Chief Complaint or Triage Note.

Among the 100 linked records with valid ICD-10-CM injury mechanism codes, the percent agreement between the two data sources on whether the ED visit was a "pedestrian" or "bicycle" crash was 54.4\% and 71.9\%, respectively. Percent agreement decreased for "traffic" and "non-traffic" designations, however. The most common V-code assigned to misclassified pedestrian/bicycle crashes in the NC DETECT ED visit data was "V87.7XXA-Person injured in a collision between other specified motor vehicles (traffic)".

Although the linkage study used data obtained from only a single Level I Trauma Center and primarily a single facility in NC DETECT, we felt that the results of this limited linkage study were generalizable to statewide NC DETECT ED visit data. For example, many facilities in NC DETECT underreport injury mechanism codes. Therefore, we added pedestrian/bicycle crash injury-related keywords to the Traffic/Non-Traffic Pedestrian/Bicycle Crash Injury case definitions (Table 1). After inclusion of these keywords, the number of identified pedestrian and bicycle crash injury-related ED visits identified in NC DETECT increased by $16.9 \%$ and $57.9 \%$ from January-June 2018 , respectively (Figure 1). 
ISDS 2019 Conference Abstracts

\section{Conclusions}

Pedestrian and bicycle crashes represent a major cause of MVC injury morbidity and mortality. Therefore, the development and evaluation of case definitions is key for the successful surveillance of these types of injuries. The inclusion of keywords can help account for some of the injury mechanism data missingness common to ED surveillance systems.

\section{Acknowledgement}

This study was supported by Project Number M3DA-18-14-03 from the NC GHSP.

Data Attribution \& Disclaimer: NC DETECT is a statewide public health syndromic surveillance system, funded by the NC DPH Federal Public Health Emergency Preparedness Grant and managed through collaboration between NC DPH and UNC-CH Department of Emergency Medicine's CCHI. The NC DETECT Data Oversight Committee does not take responsibility for the scientific validity or accuracy of methodology, results, statistical analyses, or conclusions presented.

\section{References}

1. NHTSA. Traffic Safety Facts 2015. DOT HS 812 384. Washington, DC: US Department of Transportation; 2017. https://crashstats.nhtsa.dot.gov/Api/Public/ViewPublication/812384. Accessed Sept 12, 2018.

2. Thomas L, Vann, M, Levitt D. North Carolina Pedestrian Crash Trends and Facts 2011-2015. RP 2017-42. Chapel Hill, NC: University of North Carolina Highway Safety Research Center; 2018. http://www.pedbikeinfo.org/pbcat_nc/pdf/summary_ped_facts11-15.pdf. Accessed Sept 12, 2018.

3. NCIPC. Help and Tools for Injury Data; Atlanta, GA: CDC 2018. https://www.cdc.gov/injury/wisqars/dataandstats.html. Accessed Sept 12, 2018.

4. Harmon K, Barnett C, Marshall S, Waller A. Implementing the External Cause Matrix for Injury Morbidity - North Carolina Emergency Department Data - January 2015 - May 2015. Chapel Hill, NC: Carolina Center for Health Informatics and the Injury Prevention Research Center; 2016. https://ncdetect.org/files/2017/03/ICD10CCMExternalCauseMatrixImplementation_NCSQI_201607.pdf. Accessed Sept $12,2018$. 
Figure 1. Comparison of pedestrian/bicycle crash injury-related ED visits with and without keywords: NC DETECT, January 1 - June 30, 2018

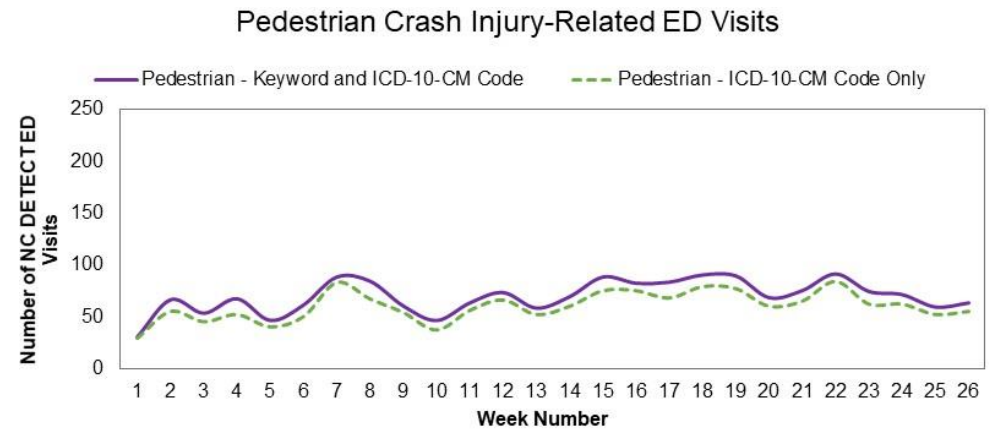

Bicycle Crash Injury-Related ED Visits

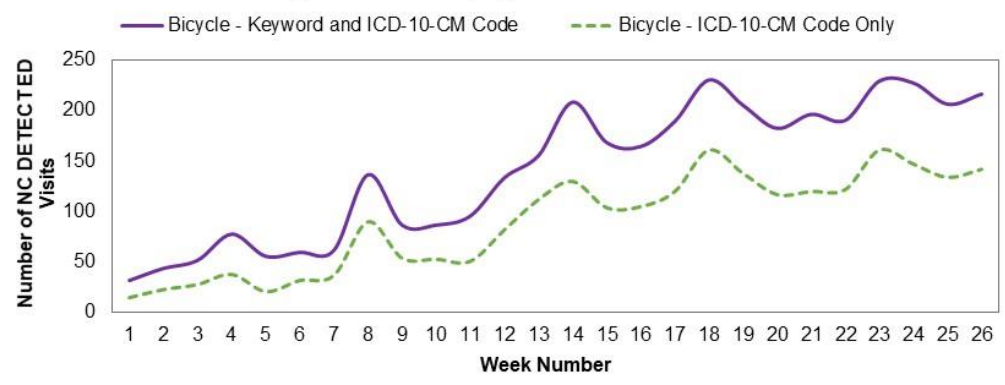

Table 1. List of pedestrian and bicycle crash injury-related keywords used in NC DETECT case definitions

\begin{tabular}{|c|c|c|}
\hline \multirow{2}{*}{ Inclusions } & \multicolumn{2}{|c|}{$\begin{array}{c}\text { Crash } \\
\text { Type }\end{array}$} \\
\hline & $\begin{array}{c}\text { Pedestrian } \\
\text { STRUCK', 'PED VS MVC', 'PEDS VS } \\
\text { MVC', 'PED VS CAR', or 'PEDS VS } \\
\text { CAR' }\end{array}$ & 'BICYCLE', 'BIKE', ' PEDAL', or 'BICYCLIST' \\
\hline Exclusions & 'MOPED', 'SCOOTER', 'PEDAL', & 'BICYCLE', or 'BIKE' \\
& \multicolumn{2}{|c|}{$\begin{array}{c}\text { MOTORCYCLIST', 'MOTOR CYCLIST', 'SCOOTER', 'MOTORCYCLE','PEDAL } \\
\text { PULSE','PEDAL EDEMA', 'PEDAL PULSES', 'MOPED','DIRT BIKE', } \\
\text { 'DIRTBIKE','MOTOR BIKE', 'MOTORBIKE', 'CAR OR BIKE', or 'PEDESTRIAN' }\end{array}$} \\
\hline
\end{tabular}

